\title{
Conocimiento y Fuentes de Información sobre las ITS/VIH, Comunicación sobre Sexo y Actitud Hacia el Uso del Preservativo en Adolescentes y Padres/Madres Salvadoreños
}

\author{
Knowledge and Sources of Information about STIS/HIV, Communication about Sex \\ and Attitude towards Condom Use in Adolescents and Parents/Mothers Salvadorans
}

\author{
Alfonso Zúñiga Fuentes ${ }^{1}$, Inmaculada Teva ${ }^{1}$ y Maria Paz Bermúdez ${ }^{1}$
}

\section{Resumen}

El objetivo general de este estudio fue examinar el conocimiento de padres/madres e hijos acerca de la prevención y la transmisión de las infecciones de transmisión sexual (ITS)/VIH, la comunicación familiar sobre la sexualidad y la actitud hacia el uso del preservativo. Participaron 326 progenitores y 326 hijos adolescentes salvadoreños. Los progenitores tenían un mayor conocimiento que sus hijos $(p=.04)$, mientras que éstos mostraban una mayor actitud positiva hacia el uso del preservativo $(p=.00)$. Los padres informaban de una mayor frecuencia de comunicación (Media padre $=11.65 ; D T=4.07$; Media madres $=11.75 ; D T=3.99$ ) de la que indicaban sus hijos (Media comunicación con el padre $=8.92 ; p=.00$; media comunicación con la madre 10.78; $p=.01)$. Estos resultados indican la necesidad de intervenciones sobre salud sexual en adolescentes latinos desde una perspectiva socio-ecológica.

Palabras clave: adolescencia, padres; sexualidad, ITS/VIH, conocimiento, fuentes de información

\begin{abstract}
The general aim of this study was to make a comparative analysis between parents and adolescents regarding knowledge about prevention and transmission of Sexually Transmitted Infections (STI) and HIV, communication about sexuality and attitude towards condom use. Participants were 326 parents and 326 adolescents from El Salvador. Results showed that parents had a higher knowledge compared to adolescents $(p=.04)$. Adolescents showed a higher positive attitude towards condom use compared to their parents $(p=.00)$. Fathers $(M e a n=11.65 ; S D=4.07)$ and mothers (Mean=11.75; $S D=3.99)$ reported a higher frequency of communication with adolescents than they did (Mean in communication with father=8.92; $p=.00$; mean in communication with mother $10.78 ; p=.01$ ). These results indicate the need on sexual health interventions in Latino teens from a socio-ecological perspective.
\end{abstract}

Keywords: adolescence, parents, sexuality, knowledge, sources of information

1 Centro de Investigación Mente, Cerebro y Comportamiento (CIMCYC). Universidad de Granada, España. Correspondencia: Inmaculada Teva. Depto. Psicología Evolutiva y de la Educación. Facultad de Ciencias de la Educación. Universidad de Granada18011 Granada, España. Tel.: +34 958243969. Correo: inmate@ugr.es 


\section{Introducción}

La adolescencia constituye una etapa de la vida en la que el individuo comienza a interactuar en múltiples situaciones vitales novedosas (Carvalho, 2015), entre ellas, la sexualidad. Con el objetivo de alcanzar una óptima salud sexual y reproductiva es necesario conocer los aspectos psicosociales relacionados con las conductas sexuales. En esta línea, la implicación parental y el conocimiento sobre el VIH y las Infecciones de Transmisión Sexual (ITS) constituyen factores esenciales en la reducción de conductas sexuales de riesgo en adolescentes (Lee, Cintron \& Kocher, 2014). Así, un mayor conocimiento sobre las ITS/VIH, una actitud positiva hacia el uso del preservativo y la existencia de comunicación entre los padres y sus hijos sobre sexualidad actuarían como factores protectores previniendo que los adolescentes se impliquen en conductas sexuales de riesgo (Castro, Bermúdez, \& Buela-Casal, 2009).

Tanto la falta de conocimientos adecuados sobre las ITS/VIH como las concepciones erróneas serían obstáculos en la adopción de conductas saludables (DiClemente, Crosby, \& Wingood, 2002). Las encuestas realizadas a jóvenes de entre 15 y 19 años de diversos países latinoamericanos indican que la mayoría de ellos ha escuchado acerca del VIH, pero al mismo tiempo no posee conocimientos consolidados sobre cómo protegerse frente al virus (DeMaría, Galárraga, Campero, \& Walker, 2009). Otros resultados muestran que a pesar de tener conocimientos adecuados, se observan diferencias en función del país y continúan persistiendo las ideas erróneas (e.g., Bermúdez et al., 2012). Con respecto a los padres, se ha observado que, en general, tienen un nivel adecuado de conocimientos sobre las ITS/VIH, aunque todavía existen ciertas concepciones erróneas que indicarían la necesidad de fortalecer las estrategias educativas centradas en ellos (Orcasita Pineda, Uribe Rodríguez, \& Valderrama Orbegozo, 2013). Concretamente, en El Salvador, se ha señalado la necesidad de mejorar el nivel conocimiento sobre las formas de prevención del VIH y su evaluación (División de Investigaciones, 2008; Zometa et al., 2007).
La comunicación entre padres e hijos adolescentes es esencial en la transmisión de conocimientos, valores y actitudes siendo la implicación de los padres un factor esencial en todas las experiencias educativas de sus hijos (Carapito, Ribeiro, \& Pereira, 2015). En el ámbito sexual, una mayor frecuencia de comunicación tendría como consecuencia que los adolescentes acudan a sus progenitores cuando necesiten información al respecto (Sales et al., 2008). Así, en una revisión reciente de estudios se concluyó que los padres que participaban en intervenciones centradas en la comunicación parental sobre el sexo, mejoraban en varios dominios de la comunicación como por ejemplo, la frecuencia, la calidad y las intenciones comunicativas (Akers, Holland, \& Bost, 2011). Otros hallazgos apuntan a que una mayor comunicación entre padres e hijos adolescentes sobre sexualidad se asocia con el incremento de actitudes positivas hacia el uso del preservativo $\mathrm{y}$, por tanto, con su uso más frecuente (Malcolm et al., 2012).

Entre los aspectos íntimamente relacionados con el nivel de conocimientos de los adolescentes sobre las ITS/VIH se encuentra la procedencia de la información sobre dichas infecciones. Así, se ha observado que cuanto mayor es el número de fuentes de información al que tienen acceso los adolescentes, mayor es el conocimiento adecuado sobre el VIH (Nguyen, Shiu, \& Peters, 2015). Cabe mencionar entre algunas de estas fuentes a los medios de comunicación, panfletos, pósters, padres, amigos, profesores, entre otras (e.g., Nwagwu, Okoye, \& Isiugo-Abanihe, 2011). En general, las principales suelen ser la familia, los amigos y la escuela (González Valcárcel, Núñez Aragón, Couturejuzon González, \& Amable Ambrós, 2008; Stern, Cooper, \& Gibbs, 2015). En un estudio realizado en El Salvador, Ruiz-Canela et al. (2012) concluyeron que, a la hora de iniciarse en las relaciones sexuales, los adolescentes tenían muy en cuenta los mensajes y actitudes de sus padres, hermanos y amigos sobre la sexualidad.

La actitud se refiere a la tendencia a responder de la misma forma ante un objeto o situación. Es de naturaleza aprendida (Ajzen \& Fishbein, 1975) y constituye un factor determinante para que la conducta finalmente se lleve a cabo (Newby, Brown, French, \& Wallace, 2013). De esta forma, 
las actitudes positivas hacia el uso del preservativo inciden en su uso habitual (e.g., Castro, Bermúdez, Buela-Casal, \& Madrid, 2011; Giménez-García, Ballester-Arnal, Gil-Llario, Cárdenas-López, \& Durán Baca, 2013). Además, las actitudes de los adolescentes sobre la sexualidad están muy influenciadas por las actitudes que existen en el seno familiar (Ferreira et al., 2013).

En síntesis, el contexto familiar resulta esencial en la prevención de las conductas sexuales de riesgo en adolescentes hispanos (Ortega, Huang, \& Prado, 2012) ya que los padres ejercen una importante influencia en las conductas de sus hijos. Dado que los valores familiares, las actitudes y los conocimientos, entre otras características, intervienen en la conducta de los hijos y en su toma de decisiones (Cederbaum \& Hutchinson, 2016), las diferencias en este tipo de variables entre padres e hijos adolescentes pueden tener una repercusión en la conducta y educación sexual de éstos últimos (e.g., Dimbuene, 2015). A su vez, existe una escasez de estudios en los que se evalúen de forma conjunta a los adolescentes y sus padres (Cordova, Huang, Lally, Estrada, \& Prado, 2014; Dimbuene, 2015).

Por tanto, se plantea el presente estudio realizado con padres/madres y sus hijos adolescentes salvadoreños, cuyos objetivos son: 1) Comparar a padres/madres y sus hijos adolescentes en el conocimiento acerca de la prevención y la transmisión de las ITS y el VIH, la comunicación sobre sexualidad y la actitud hacia el uso del preservativo; 2) Analizar en los adolescentes las fuentes de información sobre la prevención del VIH, e indagar si difieren en función del nivel de conocimiento sobre la prevención y la transmisión de las ITS y el VIH.

\section{Método}

\section{Diseño}

Se trata de un estudio descriptivo mediante encuestas.

\section{Participantes}

La muestra total estuvo compuesta por 652 participantes (326 adolescentes y 326 padres/madres). En el grupo de adolescentes, la media de edad fue de 16.82 años $(D T=0.94)$, siendo el $41.7 \%$ varones y el $58.3 \%$ mujeres. Entre ellos, el $40.6 \%$ vivía solo con uno de los progenitores; el $45.5 \%$ con los dos padres, el $1.5 \%$ vivía con su pareja, el $11.7 \%$ con familiares y el $0.6 \%$ con amigos. Todos los adolescentes acudían a centros de enseñanza secundaria laicos. En la muestra de padres/madres, la media de edad fue de 42.83 años $(D T=6.88)$. Otras características sociodemográficas de los padres/madres se incluyen en la Tabla 1.

\section{Instrumentos}

- Cuestionario de conocimiento sobre la prevención y la transmisión de las ITS/VIH (DiClemente et al., 2002; Sikkerma et al., 2000). Este instrumento consta de 16 ítems cuyo formato de respuesta es "verdadero", "falso" o "no sé". El rango de puntuaciones va desde 0 a 16 , a mayor puntuación, mayor conocimiento. El alfa de Cronbach en la muestra de adolescentes es de .73 y en la de padres de .72 .

- Actitud hacia el uso del preservativo (Wingood \& DiClemente, 1998). Consta de ocho ítems con los que se evalúa la actitud positiva hacia el uso del preservativo. Existen cinco alternativas de respuesta, que van desde "muy en desacuerdo" hasta "muy de acuerdo". El rango de puntuaciones va desde 8 hasta 40. A mayor puntuación, mayor actitud positiva hacia el uso del preservativo. El alfa de Cronbach en la muestra de adolescentes es de .71 y en la de padres de .70 .

- Escala de comunicación sobre sexo padresadolescente (Milhausen et al., 2007; Sales et al., 2008). Evalúa la frecuencia de comunicación de los adolescentes con sus padres sobre sexualidad, uso de preservativos, ITS, sida y embarazos. Consta de diez ítems, cinco sobre comunicación con el padre y cinco sobre comunicación con la madre. El formato de respuesta es una escala tipo Likert con cuatro alternativas que van desde "Nunca" a "Frecuentemente". El rango de puntuaciones en ambas subescalas oscila entre $5 \mathrm{y}$ 20. En la versión análoga para padres (con cinco de los ítems anteriores), son ellos los que informan acerca de la comunicación sobre el sexo con el hijo adolescente. A mayor puntuación mayor comunicación. El alfa de Cronbach de la subescala de comunicación con la madre en la muestra de adolescentes es de .81. En la subescala de comunicación con el padre es de .86. En la muestra de padres, el alfa de Cronbach es de .84 . 
Tabla 1. Características sociodemográficas de los padres/madres

\begin{tabular}{|c|c|c|c|}
\hline \multirow{2}{*}{\multicolumn{2}{|c|}{ Variables }} & \multicolumn{2}{|c|}{$\begin{array}{l}\text { Padres/madres } \\
(\mathrm{N}=326)\end{array}$} \\
\hline & & $\mathrm{n}$ & $\%$ \\
\hline \multicolumn{4}{|l|}{ Sexo } \\
\hline- & Varón & 65 & 20.2 \\
\hline- & Mujer & 256 & 79.8 \\
\hline \multicolumn{4}{|c|}{ Nivel de estudios } \\
\hline- & Sin estudios & 32 & 9.8 \\
\hline- & Estudios primarios & 118 & 36.3 \\
\hline- & Estudios secundarios & 111 & 34.2 \\
\hline- & Estudios tecnológicos & 21 & 6.5 \\
\hline- & Estudios universitarios & 43 & 13.2 \\
\hline \multicolumn{4}{|c|}{ Estado civil } \\
\hline- & Soltero/a & 60 & 18.4 \\
\hline- & Casado/a por la Iglesia & 109 & 33.4 \\
\hline- & Casado/a por lo civil & 71 & 21.8 \\
\hline- & Separado/a o divorciado/a & 34 & 10.4 \\
\hline- & Vive en unión libre & 34 & 10.4 \\
\hline- & Viudo/a & 14 & 4.3 \\
\hline- & Otro & 4 & 1.2 \\
\hline \multicolumn{4}{|c|}{ Situación laboral actual } \\
\hline- & En activo & 265 & 82.8 \\
\hline- & Desempleado & 55 & 17.2 \\
\hline
\end{tabular}

- Cuestionario de fuentes de información sobre cómo prevenir el VIH. Se elaboró un cuestionario ad-hoc destinado a los adolescentes para analizar cuál era la procedencia de la información sobre prevención del VIH (referida a los últimos seis meses). En primer lugar, se preguntó si habían recibido información sobre cómo prevenir el VIH en los últimos seis meses, a lo que se podía responder "si" o "no". A continuación, se solicitaba que se señalase la procedencia de la información a partir de una lista que contenía las siguientes fuentes: programas de radio $\mathrm{y} / \mathrm{o}$ televisión; anuncios en radio y/o televisión; artículos de revistas y/o periódicos; anuncios en revistas y/o periódicos; carteles publicitarios; folletos informativos; organizaciones comunitarias; asociaciones juveniles; charlas en el instituto; amigos/as/conocidos/as; familiares; tu padre; tu madre; línea de información telefónica sobre el sida o sexualidad; internet. Se podían señalar varias opciones a la vez.

Los cuestionarios de conocimiento sobre la prevención y la transmisión de las ITS/VIH, actitud hacia el uso del preservativo y comunicación sobre sexo se adaptaron lingüísticamente al castellano de la versión original en inglés. En una primera fase, se realizó el proceso de traducción-retrotraducción de los ítems al castellano. A continuación, diez jueces españoles expertos en sexualidad y adolescentes revisaron los ítems e indicaron si éstos se comprendían correctamente, o, en caso contrario, los cambios que se debían realizar. Al alcanzarse el $100 \%$ de acuerdo entre los jueces, los instrumentos fueron sometidos a evaluación por un grupo de adolescentes y otro de padres, a través de grupos focales, a los que se les preguntaba sobre su comprensión de los ítems.

\section{Procedimientos}

La realización del estudio fue aprobada por la institución académica correspondiente. A continuación se contactó y solicitó colaboración a los directores de los centros escolares que autorizaban el acceso a los adolescentes y los padres/madres. Participaron todos los centros de enseñanza secundaria de la ciudad de San Miguel. Dos investigadores llevaron a cabo el contacto con los centros y la evaluación. Se programaron reuniones con los padres de los alumnos en las que se les solicitaba su colaboración para participar en el estudio, se les explicaban los objetivos, que su participación era voluntaria y anónima y que se tratarían sus datos de forma confidencial. La mayoría aceptó hacerlo y proporcionó su consentimiento para la participación de sus hijos. Los padres respondieron los cuestionarios en la misma reunión donde se les informaba sobre el estudio y se solicitaba su colaboración. A cada cuestionario se le asignó un código que se correspondió con el 
adjudicado a su hijo/a. Los adolescentes respondieron los cuestionarios en las aulas escolares. Igualmente se les informó que su participación era voluntaria y anónima y sobre la confidencialidad de los datos.

\section{Análisis estadísticos}

Se utilizaron las pruebas $\mathrm{t}$ de Student, el análisis multivariado de la varianza (MANOVA) $y$ chi cuadrado. El estadístico t de Student se empleó para analizar las diferencias entre las medias de dos grupos (padres/madres e hijos). El MANOVA fue utilizado para minimizar los errores tipo I al analizar varias variables dependientes. La prueba chi cuadrado se empleó para la comparación de frecuencias. Los análisis estadísticos se llevaron a cabo utilizando el paquete estadístico SPSS versión 15.0 y se estableció un nivel de significación de $p \leq .05$.

\section{Resultados}

\section{Estadísticos descriptivos}

En la Tabla 2 se muestran los estadísticos descriptivos (medias y desviaciones típicas) sobre los conocimientos acerca de las ITS y el VIH, la actitud positiva hacia el uso del preservativo y la frecuencia de comunicación sobre sexo entre los miembros de la familia.

Conocimiento sobre la prevención y la transmisión de las ITS y el VIH y actitud hacia el uso del preservativo en adolescentes y padres

Se realizó un MANOVA para analizar si el grupo de adolescentes y el de padres/madres diferían en la actitud hacia el uso del preservativo y en el conocimiento acerca de la prevención y la transmisión de las ITS y el VIH. Puesto que a nivel teórico las variables sociodemográficas se relacionan con las variables analizadas (e.g., de Araújo, Teva, \& Bermúdez, 2014), el sexo, la edad y el nivel de estudios de los padres/madres se introdujeron como variables control. El modelo resultó significativo $(F(2,599)=140.74 ; p=.00$; Lambda de Wilks $=0.68$; eta al cuadrado parcial=.32). Los dos grupos diferían en la actitud hacia el uso del preservativo $(F(4,604)=12.51$; $p=.00$; eta cuadrado parcial $=.08)$ y en conocimiento sobre ITS/VIH $(F(4,604)=2.61$; $p=.04$; eta al cuadrado parcial .02). Los padres/madres tenían un mayor conocimiento sobre la prevención y la transmisión de las ITS y el VIH comparados con los hijos adolescentes mientras que éstos mostraban una mayor actitud positiva hacia el uso del preservativo (véanse las medias en las respectivas variables y en ambos grupos en la Tabla 2). No obstante, de acuerdo con Cohen (1992), los tamaños de los efectos son pequeños.

Se observó que únicamente el 18,30\% ( $n=59)$ de los adolescentes tenía un conocimiento correcto acerca de la ineficacia de la ducha vaginal para prevenir las ITS después de tener relaciones sexuales. Por otra parte, solo el $22.40 \%$ $(n=72)$ de los adolescentes sabía que el uso del preservativo con espermicida no protege frente al VIH. Únicamente el $20.50 \% \quad(n=66)$ de los adolescentes y el $27.50 \% \quad(n=87)$ de los padres/madres conocían que los preservativos de látex son mejores que los hechos de piel de oveja para prevenir el VIH.

Frecuencia de comunicación sobre sexo entre adolescentes y padres/madres

Se comparó al grupo de adolescentes con el grupo de padres y con el de madres en relación con la frecuencia de comunicación sobre el sexo (nótese que la escala de frecuencia de comunicación distinguía entre la comunicación con el padre y con la madre, respectivamente). Se observó que los padres informaban una mayor frecuencia de comunicación (Media=11.65; $D T=4.07)$ de la que indicaban sus hijos $($ Media $=8.92 ; D T=4.10)(t(347)=-4,01 ; p=.00 ; d$ de Cohen=.66). El tamaño del efecto de estas diferencias era de un grado medio. Igualmente, las madres indicaban una mayor frecuencia de comunicación con sus hijos (Media=11.75; $D T=3.99)$ de la manifestada por éstos $($ Media $=10.78 ; D T=3.88)(t(530)=-2,83 ; p=.01 ; d$ de Cohen=.24). El tamaño del efecto de estas diferencias entre madres e hijos era pequeño.

Fuentes de información sobre la prevención del $V I H$ en adolescentes en función de su nivel de conocimiento sobre la transmisión y la prevención de las ITS y el VIH

Se establecieron tres niveles de conocimiento mediante los percentiles 33 y 66 (niveles bajo y alto, respectivamente). Los percentiles intermedios constituyeron el nivel medio de conocimiento. Así, 128 adolescentes se ubicaron en un nivel bajo, 88 en un nivel medio y 106 en 
Tabla 2. Estadísticos descriptivos. Conocimiento sobre la prevención y transmisión de ITS y

VIH, actitud hacia el uso del preservativo y frecuencia de comunicación sobre sexo con el padre, con la madre y con el hijo adolescente en las muestras de adolescentes y de padres/madres

\begin{tabular}{|c|c|c|}
\hline \multirow[t]{2}{*}{ Variables } & $\begin{array}{l}\text { Adolescentes } \\
\quad(\mathrm{N}=326)\end{array}$ & $\begin{array}{l}\text { Padres/madres } \\
(\mathrm{N}=326)\end{array}$ \\
\hline & Media (DT) & Media (DT) \\
\hline Conocimiento ITS y VIH & $7.17(2.75)$ & $7.50(3.36)$ \\
\hline Actitud uso preservativo & $27.17(5.48)$ & $24.34(5.49)$ \\
\hline $\begin{array}{l}\text { Frecuencia de comunicación sobre } \\
\text { sexo con la madre }\end{array}$ & $10.78(3.88)$ & NA \\
\hline $\begin{array}{l}\text { Frecuencia de comunicación sobre } \\
\text { sexo con el padre }\end{array}$ & $8.92(4.10)$ & NA \\
\hline $\begin{array}{l}\text { Frecuencia de comunicación sobre } \\
\text { sexo con el hijo adolescente }\end{array}$ & NA & $11.65(4.07)$ \\
\hline
\end{tabular}

Nota. NA: No Aplicable.

un nivel alto. En la Tabla 3 se muestran las fuentes de información sobre la prevención del VIH en función del nivel de conocimiento sobre la transmisión y la prevención de las ITS y el VIH. Como se puede observar, los adolescentes con alto conocimiento eran los que indicaron en mayor porcentaje que habían recibido información sobre cómo prevenir el VIH en los últimos seis meses (83.8\%). Asimismo, existía un elevado porcentaje de adolescentes con bajo conocimiento que indicó que había recibido información $(75.8 \%$ ) (véase Tabla 3).

Se hallaron diferencias significativas entre los porcentajes de adolescentes con un nivel alto, medio y bajo de conocimiento que habían señalado a los familiares como fuente de información. Entre ellos, el mayor porcentaje tenía un bajo conocimiento sobre la prevención y la transmisión del VIH (43.0\%). Por otra parte, las charlas en el colegio/instituto y los folletos informativos eran las fuentes de información que se señalaron con mayor frecuencia en los tres grupos. En el total de adolescentes, la primera fuente de información eran las charlas en el colegio/instituto (78.5\%) seguida de los folletos informativos (61.8\%) e Internet (55.7\%).

\section{Discusión}

En el presente estudio se muestra que los padres/madres tienen un mayor conocimiento sobre la prevención y la transmisión de las ITS y el VIH en comparación con el de sus hijos adolescentes. No obstante, las puntuaciones medias en conocimiento en ambos grupos no son elevadas y persisten concepciones erróneas sobre las conductas para prevenir las ITS/VIH, tanto en los adolescentes como en sus padres/madres. Los adolescentes, guiados por el menor conocimiento y las concepciones erróneas que tienen sobre las ITS/VIH, podrían adoptar conductas que pongan en riesgo su salud sexual (DiClemente et al., 2002; Goldman \& Collier-Harris, 2012). Por tanto, a pesar de que se suele intervenir sobre este aspecto en los programas de prevención en población latina (Lee, Dancy, Florez, \& Holm, 2013), es necesario continuar con los esfuerzos dirigidos a incrementar los conocimientos tanto en los adolescentes como en los adultos. Aunque en los últimos años se ha observado un incremento en el gasto en prevención del VIH en El Salvador (Ministerio de Salud de El Salvador, 2014), parece ser que no se está alcanzando a toda la población, pues los avances en materia de prevención se han centrado en poblaciones de riesgo como los varones que mantienen sexo con varones y las trabajadoras sexuales (Ministerio de Salud de El Salvador, 2014). Al igual que otros organismos (e.g., División de Investigaciones, 2008), los resultados del presente estudio apuntarían a que es necesario mejorar los conocimientos sobre la transmisión y la prevención del VIH en la población adulta y adolescente. Sin embargo, al tratarse de muestras no representativas, no se pueden generalizar los resultados obtenidos. Es más, el tema sobre el conocimiento de las ITS/VIH no es solo problemático en El Salvador pues en otras 
Tabla 3. Fuentes de información sobre la prevención del VIH en función del nivel de conocimiento sobre la prevención y la transmisión de las ITS/VIH en la muestra de adolescentes

\begin{tabular}{|c|c|c|c|c|c|}
\hline $\begin{array}{l}\text { Frecuencia y fuentes de } \\
\text { información }\end{array}$ & $\begin{array}{l}\text { Conocimiento } \\
\text { bajo } \\
(n=128) \\
\mathrm{n}(\%)\end{array}$ & $\begin{array}{l}\text { Conocimiento } \\
\text { medio }(n=88)\end{array}$ & $\begin{array}{l}\text { Conocimiento } \\
\text { alto } \\
(n=106) \\
\mathrm{n}(\%)\end{array}$ & $\begin{array}{l}\text { Total } \\
(\mathrm{N}=326) \\
\mathrm{n}(\%)\end{array}$ & Chi cuadrado \\
\hline $\begin{array}{l}\text { Frecuencia: últimos } 6 \\
\text { meses, ¿has recibido } \\
\text { información sobre } \\
\text { cómo prevenir el } \\
\text { VIH? }\end{array}$ & - & - & - & - & \\
\hline $\begin{array}{l}\text { Sí } \\
\text { No }\end{array}$ & $\begin{array}{l}97(75.8) \\
31(24.2)\end{array}$ & $\begin{array}{l}58(65.9) \\
30(34.1)\end{array}$ & $\begin{array}{l}88(83.8) \\
17(16.2)\end{array}$ & $\begin{array}{l}243(75.7) \\
78(24.3)\end{array}$ & $8.34 *$ \\
\hline \multicolumn{6}{|l|}{ Fuentes información } \\
\hline $\begin{array}{l}\text { Programas de radio } \\
\text { y/o televisión }\end{array}$ & 49 (49.9) & $33(56.9)$ & $42(47.7)$ & $124(50.6)$ & 1.26 \\
\hline $\begin{array}{l}\text { Anuncios en radio } \\
\text { y/o televisión }\end{array}$ & $34(34.3)$ & $22(37.9)$ & $22(25.0)$ & $78(31.8)$ & 3.18 \\
\hline $\begin{array}{l}\text { Artículos de revistas } \\
\text { y/o periódicos }\end{array}$ & $27(27.3)$ & $18(31.0)$ & $31(35.2)$ & $76(31.0)$ & 1.38 \\
\hline $\begin{array}{l}\text { Anuncios en revistas } \\
\text { y/o periódicos }\end{array}$ & $26(26.3)$ & $10(17.2)$ & $18(20.5)$ & $54(22.0)$ & 1.93 \\
\hline Carteles publicitarios & $41(41.4)$ & $22(37.9)$ & $36(40.9)$ & $99(40.4)$ & 0.19 \\
\hline Folletos informativos & $55(55.0)$ & $35(60.3)$ & $62(70.5)$ & $152(61.8)$ & 4.80 \\
\hline $\begin{array}{l}\text { Organizaciones } \\
\text { comunitarias }\end{array}$ & $18(18.2)$ & $15(25.9)$ & $22(25.0)$ & $55(22.4)$ & 3.13 \\
\hline $\begin{array}{l}\text { Asociaciones } \\
\text { juveniles }\end{array}$ & $29(29.3)$ & $12(20.7)$ & $19(21.6)$ & $60(24.5)$ & 3.70 \\
\hline $\begin{array}{l}\text { Charlas en el } \\
\text { colegio/instituto }\end{array}$ & $82(82.0)$ & $45(77.6)$ & $66(75.0)$ & $193(78.5)$ & 1.39 \\
\hline Amigos/conocidos & $36(36.4)$ & $18(31.0)$ & $34(39.1)$ & $88(36.1)$ & 0.98 \\
\hline Familiares & $43(43.0)$ & $14(24.1)$ & $34(38.6)$ & $91(37.0)$ & $5.76^{*}$ \\
\hline Tu padre & $32(32.0)$ & $12(20.7)$ & $21(23.9)$ & $65(26.4)$ & 2.88 \\
\hline Tu madre & $45(45.0)$ & $30(51.7)$ & $30(34.1)$ & $105(42.7)$ & 4.81 \\
\hline $\begin{array}{l}\text { Línea información } \\
\text { telefónica }\end{array}$ & $21(21.2)$ & $8(14.0)$ & $13(14.8)$ & $42(17.2)$ & 3.45 \\
\hline Internet & $52(52.0)$ & $37(63.8)$ & $48(54.5)$ & $137(55.7)$ & 3.46 \\
\hline
\end{tabular}

Nota. Se podían señalar varias opciones a la vez; Porcentajes de adolescentes que señalaron dichas fuentes de información. $* p \leq .05$.

regiones del continente europeo (por ejemplo, España y Portugal) se aprecia una tendencia decreciente en el nivel de conocimientos sobre las ITS/VIH en adolescentes, que pone de manifiesto que las campañas de prevención resultan insuficientes y/o inadecuadas (Espada, Escribano, Orgilés, Morales \& Guillén-Riquelme, 2015; Ramiro, Reis, de Matos, \& Diniz, 2014).

Por otra parte, se ha mostrado que la actitud positiva hacia el uso de preservativo es mayor en los adolescentes en comparación con la de sus padres/madres. Las diferencias generacionales podrían estar en la base de este resultado. No obstante, no se han observado puntuaciones medias elevadas en ninguno de los dos grupos. Así, es necesario que los programas de prevención incidan también en promover actitudes positivas hacia el uso del preservativo tanto en los adolescentes como en sus padres, ya que éstos ejercen una gran influencia sobre sus hijos en materia de prevención de las conductas de riesgo (Ortega et al., 2012).

Con respecto a la comunicación sobre el sexo, tanto los padres como las madres manifiestan que lo hacen con mayor frecuencia de la que indican sus hijos. Este hallazgo se encuentra en la línea de otros resultados recientes (Cordova et al., 2014) de forma que se podría aprovechar para mostrar tanto a los padres como a sus hijos la existencia de esta discrepancia como punto de inicio para la mejora de la comunicación entre ellos (Cordova et al., 2014).

Se observa que no existe una alta frecuencia de comunicación sobre sexo de los adolescentes con respecto a la madre y el padre. Tampoco es muy frecuente la comunicación de los 
padres/madres con sus hijos adolescentes. Este resultado es congruente con otro del presente estudio que indica que los padres y las madres no se encuentran entre las fuentes de información más frecuentemente señaladas por los adolescentes. Aparte de la frecuencia de la comunicación sobre sexo, sería importante conocer la calidad de los contenidos de dicha comunicación, ya que a través de ella los progenitores transmiten valores, expectativas y conocimientos (de Looze, Constantine, Jerman, Vermeulen-Smit, \& ter Bogt, 2015; Jerman \& Constantine, 2010). Teniendo en cuenta los resultados obtenidos en relación con el conocimiento sobre las ITS/VIH cabría esperar que en la comunicación entre padres/madresadolescentes, no se transmiten conocimientos completamente correctos, pues las medias en tales conocimiento no son elevadas ni en los padres/madres ni en los hijos adolescentes. Estas limitaciones podrían suplirse en futuros estudios en los que se tenga en cuenta tanto la frecuencia como la calidad de la comunicación. Por otra parte, se ha sugerido que la mayoría de los padres, más que mantener un diálogo con sus hijos sobre asuntos relacionados con el sexo, se limitan a advertir sobre las amenazas y peligros (Kajula, Sheon, De Vries, Kaaya, \& Aaro, 2014). Resulta posible, por lo tanto, que las discrepancias que se han hallado entre padres e hijos sobre la frecuencia de comunicación sobre el tema se deban a ello. Se podrían llevar a cabo programas educativos en los que se forme a los padres en contenidos sobre sexualidad, ITS/VIH y en habilidades de comunicación para incrementar la frecuencia y calidad de la comunicación sobre sexo con sus hijos (Akers et al., 2011; Kajula et al., 2014; Miller et al., 2011; Poulsen et al., 2010) así como optimizar e incrementar como principales fuentes de información de los hijos a los padres y madres.

En cuanto a las fuentes de información sobre la prevención del VIH en función del nivel de conocimiento sobre las ITS/VIH, en el presente estudio se pone de manifiesto que el mayor porcentaje de adolescentes que indica que ha recibido información sobre cómo prevenir el VIH en los últimos seis meses, tiene un alto conocimiento. Igualmente, la mayoría de los adolescentes con unos niveles bajo y medio de conocimiento, también ha recibido información sobre la prevención del VIH. Se hace patente la importancia de recibir información periódicamente sobre la prevención y la transmisión de las ITS/VIH, pues se relaciona con el nivel de conocimiento. Además, la procedencia de dicha información también resulta importante. En este sentido, se encuentra que los adolescentes que en mayor porcentaje señalan a los familiares como fuente de información, tienen un bajo conocimiento sobre la prevención y la transmisión de las ITS/VIH. Asimismo, se destaca que, aunque las diferencias entre los porcentajes no son significativas (aunque se acercaban al nivel de significación estadística), aproximadamente la mitad de los adolescentes con un conocimiento bajo y medio sobre las ITS/VIH señalaron a la madre como fuente de información. Es por tanto, necesario educar especialmente en estos conocimientos a los miembros de la familia (e.g., padre, madre). En la muestra total de adolescentes, las principales fuentes de información señaladas fueron las charlas en el colegio/instituto, los folletos informativos e Internet. En esta línea, se ha concluido que a mayor variedad de fuentes de información sobre el VIH, mayor es el conocimiento adecuado al respecto, siendo el efecto superior en los adolescentes con niveles bajos de conocimiento (Nguyen et al., 2015). En un estudio realizado en El Salvador (Ruiz-Canela et al., 2012) se indicó que las principales fuentes de información de los adolescentes para aprender sobre temas relacionados con la sexualidad eran, según el siguiente orden, los padres, los amigos, los hermanos, los libros y las revistas o internet. Sin embargo, no se conocen estudios publicados en este país sobre fuentes de información acerca de la prevención del VIH en adolescentes, por lo que el presente estudio constituye una aporte a ese respecto.

Este estudio presenta algunas limitaciones. Entre ellas se encuentra que ha participado un mayor número de madres que de padres. Sería de interés evaluar en el futuro a parejas de padres y profundizar en aspectos relativos a la frecuencia de la comunicación, al tipo de información que proveen y a su calidad. Otros estudios también han puesto de manifiesto limitaciones similares (e.g., Byers \& Sears, 2012), mientras otra refiere a 
que los tamaños de los efectos encontrados son pequeños, aunque no implican que los resultados no sean relevantes. Por otra parte, estos hallazgos no se pueden generalizar a la población pues no ha participado una muestra representativa de padres y adolescentes. Se sugiere analizar en próximas investigaciones la influencia que tienen los aspectos evaluados sobre las conductas sexuales de riesgo de los adolescentes.

A pesar de las limitaciones anteriores, la aportación del presente estudio es doble. Por un lado se proporciona información de gran relevancia e interés sobre los factores analizados en población salvadoreña, sobre la que existe necesidad de contar con resultados. Por otro lado, han participado padres/madres y sus hijos adolescentes, cuando la mayoría de los estudios que se realizan se centran en uno de los dos grupos (Cordova et al., 2014). Por último, se destaca la necesidad de intervenciones sobre salud sexual en adolescentes latinos desde una perspectiva socio-ecológica, en la que se tengan en consideración múltiples niveles de influencia (individual, familiar, comunitario, entre otros) (Cardoza, Documét, Fryer, Gold, \& Butler, 2012; DiClemente, Salazar, \& Crosby, 2007).

\section{Referencias}

Ajzen, I., \& Fishbein, M. (1975). A Bayesian analysis of attribution processes. Psychological Bulletin, 82, 261-277.

Akers, A. Y., Holland, C. L., \& Bost, J. (2011). Interventions to improve parental communication about sex: A systematic review. Pediatrics, 127, 494-510. doi:10.1542/peds.2010-2194

Bermúdez, M. P., Teva, I., Ramiro, M. T., UribeRodríguez, A. F., Sierra, J. C., \& Buela-Casal, G. (2012). Knowledge, misconceptions, selfefficacy and attitudes regarding HIV: Crosscultural assessment and analysis in adolescents. International Journal of Clinical and Health Psychology, 12, 235-249.

Byers, E. S., \& Sears, H. A. (2012). Mothers who do and do not intend to discuss sexual health with their young adolescents. Family Relations, 61, 851-863.

Carapito, E., Ribeiro, M. T., \& Pereira, A. I. (2015). Questionário de envolvimento parental no jardim-de-infância (QEPJI): Estudo de validação da versão para pais. Revista Iberoamericana de Diagnóstico y Evaluación - e Avaliação Psicológica, 2, 8393.

Cardoza, V. J., Documét, P. I., Fryer, C. S., Gold, M. A., \& Butler, J. (2012). Sexual health behavior interventions for U.S. Latino adolescents: A systematic review of the literature. Journal of Pediatric and Adolescent Gynecology, 25, 136-149.

Carvalho, R. G. (2015). Desenvolvimento de projectos na adolescência: Avaliação e perspectivas sobre a intervenção psicológica. Revista Iberoamericana de Diagnóstico y Evaluación - e Avaliação Psicológica, 1, 91101.

Castro, A., Bermúdez, M. P., \& Buela-Casal, G. (2009). Variables relacionadas con la infección por VIH en adolescentes inmigrantes Latinoamericanos: Una revisión. Revista Argentina de Clínica Psicológica, 18, 227-240.

Castro, A., Bermúdez, M. P., Buela-Casal, G., \& Madrid, J. (2011). Variables psicosociales que median en el debut sexual de adolescentes en España. Revista Latinoamericana de Psicología, 43, 83-94.

Cederbaum, J. A., \& Hutchinson, M. K. (2016). Parent-child communication about abstinence and safer sex in parochial school families. Journal of HIV/AIDS \& Social Services, 15, 48-68.

Cohen, J. (1992). A power primer. Psychological Bulletin, 112, 155-159.

Cordova, D., Huang, S., Lally, M., Estrada, Y., \& Prado, G. (2014). Do parent-adolescent discrepancies in family functioning increase the risk of Hispanic adolescent HIV risk behaviors? Family Process, 53, 348-363.

de Araújo, L. F., Teva, I., \& Bermúdez, M. P. (2014). Psychological and sociodemographic variables associated with sexual risk behavior for sexually transmitted infections/HIV. International Journal of Clinical and Health Psychology, 14, 120-127.

de Looze, M., Constantine, N. A., Jerman, P., Vermeulen-Smit, E., \& ter Bogt, T. (2015). Parent-Adolescent sexual communication and its association with adolescent sexual 
behaviors: A nationally representative analysis in the Netherlands. Journal of Sex Research, 52, 257-268.

DeMaría, L. M., Galárraga, O., Campero, L., \& Walker, D. M. (2009). Educación sobre sexualidad y prevención del VIH: Un diagnóstico para América Latina y el Caribe. Revista Panamericana de Salud Pública, 26, 485-493.

DiClemente, R. J., Crosby, R. A., \& Wingood, G. M. (2002). La prevención del VIH en adolescentes. Recuperado de http://www.ibe.u nesco.org/publications/Prospects/ProspectsPdf /122s/122sdic.pdf

DiClemente, R. J., Salazar, L. F., \& Crosby, R. A. (2007). A review of STD/HIV preventive interventions for adolescents: Sustaining effects using an ecological approach. Journal of Pediatric Psychology, 32, 888-906.

Dimbuene, Z. T. (2015). Families'response to AIDS: New insights into parental roles in fostering HIV/AIDS knowledge. Journal of Biosocial Science, 47, 762-779.

División de Investigaciones (2008). El Salvador (2007): Estudio TRaC de VIH/SIDA entre Jóvenes de 15 a 24 años de San Salvador, El Salvador. Recuperado de http://www.pasca.or g/sites/default/files/07_es_trac_jov.pdf

Espada, J. P., Escribano, S., Orgilés, M., Morales, A., \& Guillén-Riquelme, A. (2015). Sexual risk behaviors increasing among adolescents over time: Comparison of two cohorts in Spain. AIDS Care, 27, 783-788.

Ferreira, M., Nelas, P., Duarte, J., Alburquerque, C., Grilo, C., \& Nave, F. (2013). Family culture and adolescent sexuality. Atención Primaria, 45, 216-222.

Giménez-García, C., Ballester-Arnal, R., GilLlario, M. D., Cárdenas- López, G., \& DuránBaca, X. (2013). Culture as an influence of the perceived risk of HIV infection: A differential analysis comparing young people from Mexico and Spain. Journal of Community Health, 38, 434-442.

Goldman, J., \& Collier-Harris, C. (2012). Schoolbased reproductive health and safety education for students aged 12-15 years in UNESCO's (2009) International Technical Guidance. Cambridge Journal of Education, 42, 445-461.
González Valcárcel, B., Núñez Aragón, E., Couturejuzon González, L., \& Amable Ambrós, Z. (2008). Conocimientos y comportamientos sobre el VIH/SIDA en adolescentes de enseñanza media superior. Revista Cubana de Salud Pública, 34. Recuperado de http://www.redalyc.org/articul o.oa?id=21419850006

Jerman, P., \& Constantine, N.A. (2010). Demographic and psychological predictors of parent-adolescent communication about sex: A representative statewide analysis. Journal of Youth and Adolescence, 39, 1164-1174.

Kajula, L. J., Sheon, N., De Vries, H., Kaaya, S. F., \& Aaro, L .E. (2014). Dynamics of parentadolescent communication on sexual health and HIV/AIDS in Tanzania. AIDS and Behavior, 18, 69-74.

Lee, Y. M., Cintron, A., \& Kocher, S. (2014). Factors related to risky sexual behaviors and effective STI/HIV and pregnancy intervention programs for African American adolescents. Public Health Nursing, 31, 414-427.

Lee, Y. M., Dancy, B., Florez, E., \& Holm, K. (2013). Factors related to sexual practices and successful sexually transmitted infection/HIV intervention programs for Latino adolescents. Public Health Nursing, 30, 390-401.

Malcolm, S., Huang, S., Cordova, D., Freitas, D., Arzon, M., Jimenez, G. L, ...Prado G. (2012). Predicting condom use attitudes, norms, and control beliefs in Hispanic problem behavior youth: The effects of family functioning and parent-adolescent communication about sex on condom use. Health Education \& Behavior, 40, 384-391. doi:10.1177/1090198 112440010

Milhausen, R. R., Sales, J. M., Wingood, G .M., Diclemente, R. J., Salazar, L. F., \& Crosby, R. A. (2007). Validation of a partner communication scale for use in HIV prevention intervention. Journal of HIV/AIDS Prevention in Children and Youth, 8, 11-33.

Miller, K. S., Lin, C. Y., Poulsen, M. N., Fasula, A., Wyckoff, S. C., Forehand, R., Long, N., \& Armistead, L. (2011). Enhancing HIV communication between parents and children: Efficacy of the Parents Matter! Program. AIDS Education and Prevention, 23, 550-563. 
Ministerio de Salud de El Salvador (2014). Informe nacional sobre el estado de situación del VIH en El Salvador en cumplimiento del plan nacional de monitoreo y evaluación del plan estratégico nacional multisectorial. Año 2013. Recuperado de http://www.salud.gob.sv /component/attachments/download/8.html

Newby, K. V., Brown, K. E., French, D. P., \& Wallace, L. M. (2013). Which outcome expectancies are important in determining young adults intentions to use condoms with casual sexual partners?: A cross-sectional study. BMC Public Health, 13, 133.

Nguyen, H., Shiu, Ch., \& Peters, C. (2015). The relationship between Vietnamese youths'access to health information and positive social capital with their level of HIV knowledge: Results from a national survey. Vulnerable Children and Youth Studies, 10, 67-78.

Nwagwu, W., Okoye, I., \& Isiugo-Abanihe, I. (2011). HIV/AIDS information source use and sexual behavior of adolescents in Ekwusigo local government area of Anambra State, Nigeria. Journal of AIDS and HIV Research, 3, 138-150.

Orcasita Pineda, L. T., Uribe Rodríguez, A. F., \& Valderrama Orbegozo, L. J. (2013). Conocimientos y actitudes frente al VIH/sida en padres de familia de adolescentes colombianos. Revista Colombiana de Psicología, 22, 59-73.

Ortega, J., Huang, S., \& Prado, G. (2012). Applying ecodevelopmental theory and the theory of reasoned action to understand HIV risk behaviors among Hispanic adolescents. Hispanic Health Care International, 10, 4252.

Poulsen, M. N., Miller, K. S., Lin, C., Fasula, A., Vandenhoudt, H., Wyckoff, S.C., Ochura, J., Obong'o C., \& Forehand, R. (2010). Factors associated with parent-child communication about HIV/AIDS in the United States and Kenya: A cross-cultural comparison. AIDS and Behavior, 14, 1083-1094.

Ramiro, L., Reis, M., de Matos, M., \& Diniz, J. (2014). Trends in adolescent sexual behavior, impact of information, and attitudes about HIV/AIDS in Portugal. Psychology, Health \& Medicine, 19, 614-624.
Ruiz-Canela, M., López-del Burgo, C., Carlos, S., Calatrava, M., Osorio, A., \& de Irala, J. (2012). Familia, amigos y otras fuentes de información asociadas al inicio de las relaciones sexuales en adolescentes de El Salvador. Revista Panamericana de Salud Pública, 31, 54-61.

Sales, J., Milhausen, R. R., Wingood, G. M., DiClemente, R. J., Salazar, L. F., \& Crosby, R. A. (2008). Validation of a parentadolescent communication scale for use in STD/HIV prevention interventions. Health Education \& Prevention, 35, 332-345.

Sikkema, K., Kelly, J., Winett, R., Solomon, L., Cargil, V., Roffman, R., ...Mercer, M. B. (2000). Outcomes of a randomized community-level HIV prevention for women living in 18 low-income housing developments. American Journal of Public Health, 90(1), 57-63.

Stern, E., Cooper, D., \& Gibbs, A. (2015). Gender differences in South African men and women's access to and evaluation of informal sources of sexual and reproductive health (SRH) information. Sex Education, 15, 48-63.

Wingood, G. M., \& DiClemente, R. (1998). Partner influences and gender-related factors associated with non condom use among adult African American women. American Journal of Community Psychology, 26, 29-51.

Zometa, C. S., Dedrick, R., Knox, M. D., Westhoff, W., Simán Siri, R., \& Debaldo, A. (2007). Translation, cross-cultural adaptation and validation of an HIV/AIDS knowledge and attitudinal instrument. AIDS Education and Prevention, 19, 231-244. 